\title{
Sacral neuromodulation therapy of the lower urinary tract: A review of the literature and unanswered questions
}

\author{
Golnaz Alemi, Vani Dandolu \\ Department of Obstetrics and Gynecology, University of Nevada School of Medicine, Las Vegas, USA \\ Email: vdandolu@unr.edu
}

Received 28 March 2013; revised 30 March 2013; accepted 9 April 2013

Copyright (C) 2013 Golnaz Alemi, Vani Dandolu. This is an open access article distributed under the Creative Commons Attribution License, which permits unrestricted use, distribution, and reproduction in any medium, provided the original work is properly cited.

\begin{abstract}
Objective: To review the evidence surrounding Sacral Neuromodulation therapy and delineate areas that will need more research. Methods: An extensive search was performed on the available literature on SNM for lower urinary tract dysfunction. Based on the results of the search, the mechanisms of action, indications, technique, and patient characteristics of therapy failures and success are presented and discussed. Results: SNM is accepted by the FDA since 1997 for the treatment of lower urinary tract dysfunction. As it is a relatively new procedure, there are variations in the technique of lead placement, generator choice, testing interval, patient selection, time to explantation, and definitions of therapy failures and successes. Conclusions: SNM is a safe and therapeutic option for the treatment of urgency-frequency syndrome, urge incontinence, and idiopathic urinary retention. However, there are multiple unanswered questions that require extensive research.
\end{abstract}

Keywords: Sacral Neuromodulation; SNM; Peripheral Nerve Evaluation; PNE; InterStim; Tined Lead Electrode; Implantable Pulse Generator; Lower Urinary Tract Dysfunction; Explantation; Urgency Frequency Syndrome; Urinary Retention; Incontinence

\section{INTRODUCTION}

Sacral neuromodulation (SNM) received approval from the US Food and Drug Administration in 1997 for the treatment of urgency-frequency syndrome, refractory urge incontinence, and idiopathic urinary retention. It has become a valuable therapeutic option for patients that have failed anticholinergic therapy, behavioral modifica- tions and pelvic floor rehabilitation. Several studies reported positive responses with SNM treatment in refractory lower urinary tract issues. However, there are also reports of treatment failures and explants. Not much is known at this time regarding the predictors of success or failure with SNM. There is limited literature that indicates higher failure in neurogenic voiding dysfunction, older populations and those with longer duration of symptoms. Several trials have evaluated InterStim Therapy and have provided the data from which surgeons decide optimal treatment protocols for their patients. However, there are many unanswered questions. It is not clear what is the most efficient and cost effective approach to select patients for SNM. Staged approach seems to be superior with close to $80 \%$ success of implanting a generator, however data is limited by small numbers. The optimum length of test phase is yet to be determined. Length of time is balanced by potential for infection at the leads. It is also not clear how long the device will stay effective and if there is deterioration in function over time either due to reaction in leads or changes in generator. The purpose of this article is to review the evidence surrounding SNM therapy and delineate areas that need more research.

\subsection{Mechanism of Action}

Sacral neuromodulation (SNM) is FDA approved for urge incontinence, non-obstructive urinary retention and urinary urgency and frequency. It is unclear how SNM works in patients with urinary dysfunction but possible explanations rely on modulating the micturition reflex pathway, rather than directly modulating the motor response of the urethral sphincter or detrusor. Normal detrusor functioning depends on sympathetic and parasympathetic neural pathways. The sympathetic nervous system allows for urinary retention and storage while the 
parasympathetic nervous system allows for detrusor contraction and bladder emptying. Supraspinal inputs from the pontine micturition center influence the micturition reflex pathway. It is hypothesized that SNM modulates signaling from the somatic and afferent nerves that control the micturition reflex pathway. Simultaneously, detrusor, sphincter and urethral vesica are affected. In patients with overactive bladder, SNM inhibits detrusor activity without affecting the strength of detrusor contractions or urethral resistance. For urinary retention, SNM suppresses the guarding reflex to decrease urethral sphincter tone and allow voiding.

\subsection{Indications}

SNM therapy is considered when the patient with OAB has exhausted conservative therapies which include lifestyle changes, pelvic floor physiotherapy, bladder training and anticholinergic medications. As of 2013, SNM has FDA approval for overactive bladder (including urgency and frequency with or without urgency incontinence), urinary retention and fecal incontinence. Clinical benefit has been documented in cases of interstitial cystitis, and chronic pelvic pain. This article focuses on SNM use pertaining to lower urinary tract.

\section{PROCEDURE AND RATIONALE}

Sacral neuromodulation can be accomplished either as a staged implantation with tined leads or a peripheral nerve evaluation (PNE) with temporary electrodes followed by full system implantation. The peripheral nerve evaluation is done as an outpatient procedure using a monopolar electrode. After correct placement of the electrode in the sacral foramina, the temporary electrode is attached to the skin with adhesives for the duration of the 4 to 7 day test period. If the patient has a successful test phase, then the temporary electrode is removed and the patient is taken to the operating room for implantation of the permanent tined lead and the implantable pulse generator (IPG).

The staged technique consists of an initial test stage (acute and subchronic neuromodulation) and then an implantation stage (chronic neuromodulation). In contrast to PNE, the staged technique uses quadripolar tined leads that contain an intrinsic retention mechanism. Prior to the introduction of the self-anchoring "tined" lead in 2002, the older leads required implantation during an open surgical procedure with the leads being secured to the sacral periosteum. The introduction of tined leads allowed for percutaneous placement under radiological guidance. The use of tined leads enabled physicians to directly evaluate sensory responses to stimulation while the patient was under local anesthesia. Other advantages included shorter operative time and post operative re- covery time, less infection and less pain. More importantly, tined leads have reduced the incidence of lead migration.

The patient is placed in prone position with visualization of the anal region and feet to assess reflex response. Either general or local anesthesia can be used but muscle relaxants should be avoided in the case of general anesthesia. Localization of the sacral nerves S2, S3 or S4 are identified with or without the aid of imaging, and a needle is inserted in the sacral foramen at an angle of about 60 degrees. Medial insertion of the needle improves the chance of obtaining optimal motor and sensory response of the anus and pelvic floor [1]. Usually, S2, S3 and S4 are tested bilaterally to identify the best location for electrode placement. If fluoroscopy is to be used, then posterior-anterior fluoroscopy can identify the sacral foramina while lateral fluoroscopy can identify the depth of needle insertion. For those patients with suboptimal anatomy or body habitus, computed tomography can be used to assist needle and electrode positioning [2].

Most commonly, the S3 foramen is targeted because it provides the best motor and sensory response to stimulation. The S3 foramen is localized halfway between the coccyx tip and the last spinous process of the lumbar spine. Govaert et al. observed similar success rates between S3 and S4 [3].

The needle is connected to an external stimulator and low amplitude electrical currents are applied. Correct positioning of the leads is confirmed by assessing motor and sensory responses to stimulation. Typical responses include contraction of the levator ani muscle, a tapping or vibrating sensation in the vaginal or anal region, and dorsiflexion of the great toe on the ipsilateral side of the test stimulation. Additionally, correct position of the leads can be confirmed by fluoroscopy. Once correct placement is confirmed, electrode leads are inserted through the needle and the needle removed.

The test period should last a minimum of three days and typically 1 - 2 weeks. The patient is required to keep a voiding diary before and during the test stimulation period. If the patient has a 50\% improvement of symptoms both subjectively and objectively then she is eligible for a stage 2 procedure. Parameters used to evaluate the clinical effect differ depending of the nature of the initial complaint. In patients with chronic urinary retention, values assessed include reduction in the volume at catheterization and increase in voided volume. For those with OAB dry, the most important parameters include the voided volume per void and voiding frequency. Lastly, in patients with OAB wet, the number of leakages per day and the number of pads used per day are evaluated. Patients with positive response then undergo stage 2 where pulse generator is implanted in a subcutaneous pocket in the lower abdomen or buttock. 
The optimal stimulation setting for the implantable neurostimulator (INS) is chosen by assessing the sensory response to different settings. The stimulation setting that gives the best sensory response at the lowest amplitude is optimal. The optimal pulse rate is generally set between 10 and $16 \mathrm{~Hz}$ and the pulse width is set at $210 \mathrm{~ms}$. It is unclear whether different pulse rates and pulse widths have different treatment efficacies. Although patients are advised to keep the INS on at all times, they are given a remote control to turn the INS on or off when necessary and alter the stimulation amplitude.

\subsection{Patient Follow-Up}

Patients are scheduled for follow up after implantation at six weeks, six months and yearly thereafter. Patients should be assessed for symptom relief, pain, patient comfort and compliance with SNS. The INS should be checked for proper impedance levels and patient compliance with stimulation settings. If the impedance is less than 50 Ohms or more than 4000 Ohms, then there may be damaged leads. Damaged or migrated leads can result in loss of efficacy and, thus, therapy failure. Leads can be replaced or new leads can be inserted in the contralateral sacrum. Pain can be located at the implantation site or the site of perceived sensation. If the stimulator is turned off and the pain persists, then the pain is at the implantation site and likely physical discomfort. However, if the pain decreases, then it is stimulation related. Pain relief can be achieved by modifying INS settings, repositioning the INS or adjusting lead placement. Approximately thirty-three percent of patients have required surgical revision and ten percent have needed permanent removal [4].

\subsection{Staged Implantation vs PNE}

The first study of staged implantation reported a $60 \%$ to $90 \%$ improvement in treatment response when using the permanent electrode in a cohort of patients with a negative PNE result [5]. Subsequent studies compared PNE and staged implantation and used the progression rate to IPG implantation as the determinant of success. In 2004, Everaert reported that patients with positive PNE had better treatment success with staged implantation after two years [6]. Bannowsky et al. conducted a study in patients with voiding dysfunction who underwent bilateral PNE or staged tined leads implantation. They found that $82 \%$ of those receiving staged tined leads progressed to IPG placement, while $47 \%$ of PNE patients progressed [7]. Similarly, a study evaluated 30 patients with refractory urge incontinence who were randomized to either the staged or PNE technique. $88 \%$ of staged patients progressed to IPG compared to $46 \%$ in the PNE group [8].
The differences in IPG implantation rates between PNE and staged techniques are not clearly understood. However, the quadripolar leads have been shown to have greater variety of programming options and stimulation patterns that can be manipulated to benefit the patient. The success of staged implantation is likely due to the use of the permanent electrodes for evaluation. In fact, three different trials found that permanent electrodes doubled the treatment response rate of SNM [9-11]. Additionally, some patients who fail a PNE test will pass a staged trial [12]. A review by Baxter reported that IPG implantation rates approached $40 \%$ to $50 \%$ in patients undergoing $\mathrm{PNE}$ and $70 \%$ to $90 \%$ in those undergoing staged trials [13]. Furthermore, the rate of lead migration that is seen in PNE is not seen in tined leads. Thus, there is more effective stimulation of the sacral nerves [11].

To date, there are few cost-benefit analyses comparing staged implantation to PNE. A study by Nikolavsky et al. showed that in patients with Blue Cross/Blue Shield insurance the single stage procedure would be more cost effective than a two-stage implantation. In patients with Medicare, the cost differences were not seen [14]. Other studies have stated that Medicare reimburses the office-based PNE better over staged implantation [13].

\subsection{Bilateral vs Unilateral SNM}

There is no consensus on the placement of unilateral or bilateral tined leads. Sheepens et al. reported that there was no advantage of bilateral lead placement, although some patients with nonobstructed urinary retention required bilateral SNM for success [15]. Similarly, Marcelissen et al. found limited success with bilateral stimulation in patients who had previously failed unilateral stimulation [16]. Overall, unilateral SNM is effective in many patients and patients who fail treatment may be offered bilateral evaluation [17].

\section{PATIENT CHARACTERISTICS OF SUCCESS}

There is no consensus regarding patient characteristics that should be considered contraindications for SNM. Amundsen et al. concluded that older age, the presence of neurological conditions, spinal cord disease, the presence of three or more chronic conditions, and longer duration of complaints are negatively associated with SNS success [18]. In contrast, Wallace's study of 33 patients with underlying neurologic dysfunction showed a 93\% satisfaction rate with SNM therapy [19]. A study in 2009 by White et al. followed 221 patients for three years and indicated that predictors of adverse events included a history of interstitial cystitis, pain clinic patients, and any type of trauma post implantation of InterStim [20]. Interestingly, a delay in IPG implant after successful PNE 
test adversely affected the success rate of SNM. Three studies reported that the longer duration of follow up has more risks of complications [20-22].

Additionally, two studies found that patients who had a change in their body mass index post implantation had more complications than those who did not have significant weight fluctuations $[20,23]$. However, obesity itself is not considered a risk factor for SNS failure and a 2012 study by Levin et al. concluded that obesity should not be a contraindication for InterStim therapy [24].

Intraoperative predictors of success include motor and sensory response to stimulation during lead placement. A study in 2006 concluded that sensory response was not a reliable indicator of success [25]. A similar study in 2011 concluded that intraoperative sensory testing is not as predictive of success as is motor response testing [26].

\subsection{Lead Revisions, Removals, Explants}

Complications with SNS are reported as adverse events that encompass change in clinical efficacy, pain, wound complication, infection, lead migration, and device failure. Many problems can be managed conservatively but some require surgical intervention. Van Kerrebroeck's multicenter trial reported that $67 \%$ of 152 implanted patients had adverse events. Two hundred twenty one adverse events were recorded [21]. Sixty patients required surgical intervention and 16 required permanent explantation. Reasons for surgical intervention included device exchanges, IPG or lead repositioning and wound care.

Another multicenter trial reported a 33\% surgical revision rate in 219 implanted patients [27]. Cited reasons for adverse events included pain at the IPG site, lead migration, new pain, infection, transient electrical shock, adverse changes in bowel or voiding function, device malfunctions, change in menses, skin irritation and nerve injury. The advent of tined leads in 2002 has minimized the adverse events of SNM. Siddiqui et al. systematically reviewed the literature from 1980 to September 2008 and identified five studies that addressed issues of pain, lead migration and infection with tined leads. It was found that $3 \%$ to $16 \%$ of patients required surgical revision [28]. Explantation due to lack of efficacy was performed in $6 \%$ of patients while $5 \%$ to $11 \%$ were explanted due to infection. They concluded that the use of tined leads result in considerably lower revision rates. Yazdany had an explantation rate of $25 \%$ and a surgical revision rate of $24 \%$ [29]. Yazdany reported the average time to explantation was 2.8 years.

\subsection{Predictors for Explantation}

Yazdany et al. attempted to identify patient characteristics that would predict explantation [29]. Previous studies have reported explant rates of 9\% [27]. However,
Yazdany's study of 58 patients reported a considerably higher explant rate of $25 \%$. Their reasons for explantation were similar to other published studies but also included surgeon preference as an important criterion. They were able to exclude history of hypertension, diabetes, incontinence, BMI, age, prolapse surgery, hysterectomy, menopause, duration of symptoms and type of lead as predictors of device explant. Patients with worse baseline quality of life questionnaire scores on the Incontinence Impact Questionnaire (IIQ-7) were more likely to have device explantation.

Rates of adverse events, surgical revision and explantation thus vary from study to study, some variation is attributable to inconsistent use of new technology, inconsistent patient follow up with the same physician, patient compliance and surgeon preference of revision versus explantation. Thus, counseling patients about the risks and benefits of SNS becomes heavily dependent on the physician's understanding of the literature and personal biases.

\subsection{Long Term Success Rate}

Most studies defined success of treatment as the percentage of patients who had more than 50\% improvement in key voiding diary variables at last follow up visit. Siegel's multicenter, prospective trial of 41 patients with urge incontinence, 29 patients with urgency frequency, and 42 patients with urinary retention reported persistant improvement of symptoms after 18 to 36 months of InterStim usage [27]. Similarly, van Kerrebroeck's multicenter, prospective trial demonstrated a 5-year post-implantation success rate of $68 \%$ for urinary urge incontinence, $56 \%$ for urgency frequency, and $71 \%$ for urinary retention [21]. Siddiqui analyzed the three most representative studies to date and showed that 234 implanted patients had significant improvement in mean incontinent episodes per day. A five-year follow up of those patients reported that $54 \%$ of patients maintained improvement [28].

Other studies have reported that $75 \%$ of patients not improved by conservative options such as medications and biofeedback therapy can expect a therapy response with InterStim treatment [30].

\section{CONCLUSIONS}

Sacral neuromodulation has become a valuable treatment option for chronic lower urinary tract voiding dysfunction. Multiple factors are integral to the success of SNM therapy including but not limited to patient selection, surgical technique, patient compliance, insurance status, and surgeon preference. Furthermore, advancements in technology are improving rates of revision, explantation and patient satisfaction. Regardless, our understanding of 
how neuromodulation works is still incomplete. And, despite of the significant number of patients undergoing SNM, there are many unanswered questions.

Patient Selection: While certain patient characteristics have proven to be predictors of SNM success, it is still unclear how comorbidities influence success and what modifications would be effective in combating those issues. Furthermore, as SNM is being expanded to the pediatric population better guidelines are needed to define optimal treatment. In addition, algorithms for patient follow up and evaluations are needed. What are the predictors of success? Are there urodynamic predictors of success? Are there patient characteristics that predict success or failure? What is the efficacy in diabetics, multiple sclerosis or other neurological conditions? How effective is the devise in older populations, obese patients and those with long standing symptoms?

Staged Procedures: More data is needed to compare the benefits and risks of a two-stage sacral nerve stimulation procedure versus out patient PNE followed by full system implantation. Response rates after PNE need to be studied and ultimate chance of patient getting generator with the two approaches needs to be determined. Provider and facility costs with these two approaches need to be taken into consideration as well. Is PNE worth offering as the overall rate of getting generator is low in patients that start of with PNE compared to direct stage 1? Are there activity restrictions and recommendations, better adhesive leads to improve the efficacy of PNE?

Is the overall cost burden related to procedure less with direct stage 1 compared to PNE? What is the ideal test interval between PNE and stage 2? Is it between stage 1 and stage 2? Is there difference between the two protocols?

Lead Placement/IPG Choice: Variations of tined lead placements need to be explored. It is unclear if unilateral SNM is as effective as bilateral SNM and whether the choice of sacral foramina (S3 vs.S4) impact success rate. Is there evidence to examine effect of lead placement at S2 or S4 in patients that fail S3 lead placement?

Therapy Time/Failures: It is unclear how long a patient can stay on SNM therapy before it starts to fail. Whether there are patient and/or device indications that can signal decreasing efficacy need to be evaluated. What is the long-term success of SNM? Does the performance of device or leads decline over time? If a patient fails SNM, what are the next steps in therapy?

Alternatives to SNM: Are there viable alternatives to SNM? Would peripheral nerve stimulation via the pudendal nerve or the posterior tibial nerve and botox injection into bladder be good options in certain patient populations? Is there concrete evidence for bilateral lead placement?

Future multicenter trials will help to answer these questions and allow surgeons to provide the best care possible. A working group of high volume users of SNM, and a prospective registry will also help to answer some of these questions.

\section{REFERENCES}

[1] Spinelli, M. (2008) Latest technologic and surgical developments in using InterStim therapy for sacral neuromodulation: Impact on treatment success and safety. European Urology, 54, 1287-1296.

doi:10.1016/j.eururo.2008.01.076

[2] Amoroso, L., Pelliccioni, G., Ghiselli, R., et al. (2005) Sacral-neuromodulation CT-guided. La Radiologia Medica, 9, 421-429.

[3] Govaert, B. (2009) Can sensory and/or motor reactions during percutaneous nerve evaluation predict outcome of sacral nerve modulation? Diseases of the Colon \& Rectum, 52, 1423-1426. doi:10.1007/DCR.0b013e3181a91241

[4] Vasdev N. (2010) The surgical management of the refractory overactive bladder. Indian Journal of Urology, 26, 263-269. doi:10.4103/0970-1591.65402

[5] Janknegt, R.A., Weil, E.H. and Eerdmans, P.H. (1997) Improving neuromodulation technique for refractory voiding dysfunctions: Two stage implant. Urology, 49, 358362. doi:10.1016/S0090-4295(96)00506-7

[6] Everaert, K., Kerckhaert, W., Caluwaerts, H., et al. (2004) A prospective randomized trial comparing the 1-stage with the 2-stage implantation of a pulse generator in patients with pelvic floor dysfunction selected for sacral nerve stimulation. European Urology, 45, 649-654. doi:10.1016/j.eururo.2003.11.015

[7] Bannowsky, A., Wefer, B., Braun, P., et al. (2008) Urodynamic changes and response rates in patients treated with permanent electrodes compared to conventional wire electrodes in the peripheral nerve evaluation test. World Journal of Urology, 26, 623-626. doi:10.1007/s00345-008-0307-7

[8] Borawski, K., Foster, R., Webster, G., et al. (2007) Predicting implantation with a neuromodulator using two different test stimulation techniques: A prospective randomized study in urge incontinent women. Neurourology and Urodynamics, 26, 14-18. doi:10.1002/nau.20332

[9] Spinelli, M. and Sievert, K.D. (2008) Latest technologic and surgical developments in using InterStim therapy for sacral neuromodulation: impact on treatment success and safety. European Urology, 54, 1287-1296. doi:10.1016/j.eururo.2008.01.076

[10] Peters, K.M., Carey, J.M. and Konstandt, D.B. (2003) Sacral neuromodulation for the treatment of refractory interstitial cystitis: Outcomes based on technique. International Urogynecology Journal, 14, 223-228. doi:10.1007/s00192-003-1070-3

[11] Hauck, E.F., Schwefer, M., Wittkowski, W. and Bothe, H.W. (2009) Measurements and mapping of 282,420 nerve fibers in the S1-5 nerve roots. Journal of Neurosurgery: Spine, 11, 255-263. 


\section{doi:10.3171/2009.3.SPINE17684}

[12] Sutherland, S.E., Lavers, A., Carlson, A., et al. (2007) Sacral nerve stimulation for voiding dysfunction: One institution's 11-year experience. Neurourology and Urodynamics, 26, 19-28. doi:10.1002/nau.20345

[13] Baxter, C. and Kim, J. (2010) Contrasting the percutaneous nerve evaluation versus staged implantation in sacral neuromodulation. Current Urology Reports, 11, 310-314. doi:10.1007/s11934-010-0128-2

[14] Nikolavsky, D., Killinger, K., Boura, J. and Peters, K. (2011) Comparison of patients undergoing a two stage sacral nerve stimulation procedure: Is there a cost benefit for a single-stage procedure? International Urology and Nephrology, 43, 997-1002. doi:10.1007/s11255-011-9919-8

[15] Sheepens, W.A., De Bie, R.A., Weil, E.H. and Van Kerrebroeck, P.E. (2002) Unilateral versus bilateral sacral neuromodulation in patients with chronic voiding dysfunction. The Journal of Urology, 168, 2046-2050. doi:10.1016/S0022-5347(05)64292-9

[16] Marcelissen, T.A., Leong, R.K., Serroyen, J., et al. (2011) The use of bilateral sacral nerve stimulation in patients with loss of unilateral treatment efficacy. The Journal of Urology, 185, 976-980. doi:10.1016/j.juro.2010.10.065

[17] Amend, B., Khalil, M., Kessler, T.M. and Sievert, K.D. (2011) How does sacral modulation work best? Placement and programming techniques to maximize efficacy. Current Urology Reports, 12, 327-335. doi:10.1007/s11934-011-0204-2

[18] Amundsen, C., Romero, A.A., Jaminson, M.G. and Webster, G.D. (2005) Sacral neuromodulation for intractable urge incontinence: are there factors associated with a cure? Urology, 66, 746-750. doi:10.1016/j.urology.2005.04.031

[19] Wallace, P.A., Lane, F.L. and Noblett, K.L. (2007) Sacral nerve neuromodulation in patients with underlying neurologic disease. American Journal of Obstetrics \& Gynecology, 197, 96.e1-e5.

[20] White, W.M., Mobley, J.D., Doggweiler, R., DobmeyerDittrich, C. and Klein, F.A. (2009) Incidence and predictors of complications with sacral neuromodulation. Urology, 73, 731-735.

[21] Van Kerrebroeck, P.E., Van Voskuilen, A.C., Heesakkers, J.P., et al. (2007) Results of sacral neuromodulation ther- apy for urinary voiding dysfunction: outcomes of a prospective, worldwide clinical study. Journal of Urology, 178, 2029-2034. doi:10.1016/j.juro.2007.07.032

[22] Datta, S.N., Chaliha, C., Singh, A., et al. (2008) Sacral neurostimulation for urinary retention: 10-year experience from one UK Centre. BJU International, 101, 192196.

[23] Nold, C.J. and McLennan, M.T. (2007) Spontaneous extrusion of sacral nerve implant secondary to massive weight loss. International Urogynecology Journal and Pelvic Floor Dysfunction, 18, 105-107. doi:10.1007/s00192-006-0069-y

[24] Levin, P.J., Wu, J.M., Siddiqui, N.Y. and Amundsen, C.L. (2012) Does obesity impact the success of an InterStim test phase for the treatment of refractory urge urinary incontinence in female patients? Female Pelvic Medicine \& Reconstructive Surgery, 18, 243-246. doi:10.1097/SPV.0b013e31826150fe

[25] Cohen, B.I. (2006) Predictors of success for first stage neuromodulation: Motor versus sensory response. Journal of Urology, 175, 2178-2180. doi:10.1016/S0022-5347(06)00315-6

[26] Peters, K. (2011) Is sensory testing during lead placement crucial for achieving positive outcomes after sacral neuromodulation? Neurourology and Urodynamics, 30, 14891492. doi:10.1002/nau.21122

[27] Siegel, S.W., Catanzaro, F., Dijkema, H.E., et al. (2000) Long term results of a multicenter study on sacral nerve stimulation for treatment of urinary urge incontinence, urgency-frequency, and retention. Urology, 56, 87-91. doi:10.1016/S0090-4295(00)00597-5

[28] Siddiqui, N.Y., Wu, J.M. and Amundsen, C.L. (2010) Overactive bladder: A systematic review. Neuro and Urod. 29:S18-23. doi:10.1002/nau.20786

[29] Yazdany, T., Bhatia, N. and Nguyen, J. (2011) Determining outcomes, adverse events, and predictors of success after sacral neuromodulation for lower urinary disorders in women. International Urogynecology Journal, 22, 1549-1554. doi:10.1007/s00192-011-1512-2

[30] Aboseif, S., Tamaddon, K., Chalfin, S., Freedman, S. and Kaptein, J. (2002) Sacral neuromodulation as an effective treatment for refractory pelvic floor dysfunction. Urology, 60, 52-56. doi:10.1016/S0090-4295(02)01630-8 